\title{
Vitamin D deficiency in patients with Graves' disease: probably something more than a casual association
}

\author{
Mario Rotondi • Luca Chiovato
}

Received: 29 June 2012 / Accepted: 25 July 2012/Published online: 25 August 2012

(C) Springer Science+Business Media, LLC 2012

In addition to its established role in calcium metabolism and skeletal homeostasis, vitamin D was recently identified as a factor playing a role in both innate and adaptive immunity (reviewed in Ref. [1]). The vitamin D receptor is expressed on monocytes and activated lymphocytes and the biologically active metabolite of vitamin D, i.e., 1,25-dihydroxyvitamin D3 $\left[1,25(\mathrm{OH})_{2} \mathrm{D}_{3}\right]$, does modulate $\mathrm{T}$ cell responses [1]. Studies in animal models of autoimmune diseases consistently showed that the administration of $1,25(\mathrm{OH})_{2} \mathrm{D}_{3}$ produces selective immunosuppressant effects, as assessed by its ability to either prevent or markedly suppress experimental autoimmune encephalomyelitis, rheumatoid arthritis, systemic lupus erythematosus, type 1 diabetes mellitus, and inflammatory bowel disease [2]. Currently available clinical studies report a high prevalence of vitamin $\mathrm{D}$ deficiency in patients with several autoimmune diseases, including multiple sclerosis, rheumatoid arthritis, type 1 diabetes mellitus, and Crohn's disease [3]. With specific regard to autoimmune thyroid diseases (AITD), available data remain controversial. The administration of $1,25(\mathrm{OH})_{2} \mathrm{D}_{3}$ had little effect in reducing the severity of experimental autoimmune thyroiditis induced by immunizing CBA mice with thyroglobulin [4]. In a model of experimental Graves' disease in which mice were immunized with adenovirus encoding for the TSH-receptor gene, BALB/cJ and C57BL/6J mice receiving either a sufficient or a deficient vitamin $\mathrm{D}$ diet developed comparable levels of TSH binding inhibitory and thyroid stimulating antibodies [5]. The IgG subclasses of

\footnotetext{
M. Rotondi · L. Chiovato $(\square)$

Unit of Internal Medicine and Endocrinology, Fondazione

Salvatore Maugeri I.R.C.C.S., Laboratory for Endocrine

Disruptors and Chair of Endocrinology, University of Pavia,

Via S. Maugeri 10, 27100 Pavia, Italy

e-mail: luca.chiovato@fsm.it
}

antibodies directed to the TSH-receptor were also similar in mice of either strain, irrespective of the treatment with a vitamin D-deficient or vitamin D-sufficient diet [5]. However, as argued by the authors, it should be considered that in this experiment mice fed a vitamin $\mathrm{D}$ deficient diet, in spite of having nearly undetectable serum levels of $25(\mathrm{OH}) \mathrm{D}$, displayed low but detectable concentrations of $1,25(\mathrm{OH})_{2} \mathrm{D}$.

When human studies are taken into account, a high prevalence of vitamin D deficiency was reported both in patients with chronic autoimmune thyroiditis and in those with Graves' disease [6, 7].

In this issue of Endocrine, Yasuda et al. [8] measured the serum levels of $25(\mathrm{OH})$ vitamin $\mathrm{D}$ in 26 female patients with newly onset Graves' disease and in 46 healthy female controls. Vitamin D deficiency was diagnosed when laboratory results indicated a serum level below $15 \mathrm{ng} / \mathrm{ml}$. The authors reported significantly lower levels of vitamin D and a greater prevalence of vitamin D deficiency in Graves' patients as opposed to healthy controls. A significant inverse relationship was found between the circulating concentrations of vitamin D and thyroid volume. This observation would be in line with the finding by Vilarrasa et al. [9] who reported an inverse association between body mass index (which is positively associated with thyroid volume) and circulating $25(\mathrm{OH})$ vitamin $\mathrm{D}$. On the other hand, Yasuda et al. [8] found no association between the serum levels of $25(\mathrm{OH}) \mathrm{D}_{3}$ and TSH-, FT3-, FT4-, or TSHreceptor antibodies. Although the results of this study are limited by the small number of investigated patients and by its cross-sectional design, some specific comments can be drawn. First, a large Graves' goiter is associated with a higher rate of relapsing hyperthyroidism after stopping treatment with anti-thyroid drugs [10]. Thus, Graves' patients bearing large goiters and being less prone to be successfully cured with anti-thyroid drugs, would more 
likely have low levels of vitamin D. Second, the lack of a significant association between low vitamin D and high serum levels of TSH-receptor antibodies would indirectly support the concept that, rather than affecting the immune response, vitamin D deficiency might influence the outcome of Graves' disease through a direct effect on the thyroid gland. Several direct effects of vitamin D on the thyroid have been previously described, including a dosedependent inhibition of iodide uptake and growth induced by TSH in FRTL5 cells [5].

The above reported data imply that the role played by vitamin D in Graves' disease is still an open and intriguing issue. Most current evidence suggests a higher prevalence of vitamin D deficiency in patients with Graves' disease. However, the existence of an association, even when supported by strong statistical significance, does not automatically imply that a causal relationship does exist. Several issues should be clarified for a better understanding of the role played by vitamin $\mathrm{D}$ in thyroid autoimmunity and in Graves' diseases. A main one involves the production of the active metabolite of vitamin $\mathrm{D}$ by $25(\mathrm{OH}) \mathrm{D}_{3} 1$ - $\alpha$-hydroxylase at the tissue level. This rate limiting enzyme has been recently found in several extra-renal tissue, thus raising attention on the local production of active vitamin $\mathrm{D}$. The production of active vitamin $\mathrm{D}$ within the thyroid gland has been investigated, but the evidence for this phenomenon remains controversial. In only one study addressing this issue, a positive immunohistochemical staining for $1-\alpha$ hydroxylase was found in most samples from papillary thyroid cancers, but not in normal follicular thyroid cells [11]. Moreover, real-time quantitative RT-PCR did not show differences in the expression of $1-\alpha$-hydroxylase in papillary thyroid cancers as compared with normal thyroid tissue [11]. To further complicate the issue, the intra-thyroidal cytokine milieu could modulate the biological activity of 1- $\alpha$-hydroxylase, thus affecting the local concentrations of active vitamin D. In vitro, interferon (IFN)- $\gamma$ was shown to mediate the enzymatic conversion of 25 $(\mathrm{OH}) \mathrm{D}$ to $1,25(\mathrm{OH}) \mathrm{D}$ by increasing $1-\alpha$-hydroxylase activity in isolated immune cells [12]. However, there are no in vivo data supporting such a regulation of active vitamin $\mathrm{D}$ production by IFN- $\gamma$.

From a clinical point of view, two questions should be answered in order to understand the role of vitamin D in Graves' disease. First, we should know whether vitamin D deficiency favors the onset of the disease. Second, it would be important to clarify whether the correction of vitamin D deficiency has any beneficial therapeutic effect in patients with Graves' disease. No currently available data in humans provide straightforward answers to these questions. However, a few experimental data should be mentioned. First, BALB/cJ mice given a vitamin D deficient diet were more likely to develop persistent hyperthyroidism following three immunizations with TSH-receptor as opposed to their countermates receiving adequate vitamin D supply [5]. Second, a vitamin D analog, namely elocalcitol was shown to inhibit both the production of Th-1 and Th-17 T-helper cell cytokines, thus shifting the immune response toward a Th-2 phenotype, and the secretion of the chemokine CXCL10, which is induced by IFN- $\gamma$ in human thyroid cells [13]. CXCR-3 binding chemokines, mainly CXCL10, play a role in the pathogenesis of thyroid autoimmunity and Graves' disease [14] and their neutralization was recently proposed as novel therapeutic strategy in AITD (reviewed in Ref. [13]). Clinical intervention studies do not provide enough evidence supporting the therapeutic effect of vitamin D administration in patients with Graves' disease. Only one such study is available in which patients with Graves' disease were randomized to receive methimazole alone or methimazole + vitamin D3 $(1,25)-\mathrm{OH}$ [15]. The restoration of euthyroidism was achieved more rapidly in those patients receiving the combined treatment. However, it should be noted that study group was small, the assay employed for detecting TSH-receptor antibodies had a low sensitivity, vitamin D serum levels were not measured, and other factors known to influence the clinical outcome of Graves' hyperthyroidism were not taken into account [15]. Thus, firm conclusion on the beneficial effect of vitamin D in Graves' patients cannot be drawn from this study.

Taking into account the above reported considerations, it appears that studies specifically designed at evaluating the role of vitamin D deficiency and the effect of its correction in patients with Graves' disease are needed before firm conclusions can be formulated.

Conflict of interest The authors have nothing to disclose.

\section{References}

1. J.S. Adams, M. Hewison, Unexpected actions of vitamin D: new perspectives on the regulation of innate and adaptive immunity. Nat. Clin. Pract. Endocrinol. Metab. 4, 80-90 (2008)

2. H.F. Deluca, M.T. Cantorna, Vitamin D: its role and uses in immunology. FASEB J. 15, 2579-2585 (2001)

3. M.F. Holick, Vitamin D deficiency. N. Engl. J. Med. 357, 266-281 (2007)

4. C. Fournier, P. Gepner, M. Sadouk, J. Charreire, In vivo beneficial effects of cyclosporin A and 1, 25-dihydroxyvitamin D3 on the induction of experimental autoimmune thyroiditis. Clin. Immunol. Immunopathol. 54, 53-63 (1990)

5. A. Misharin, M. Hewison, C.R. Chen, V. Lagishetty, H.A. Aliesky, Y. Mizutori, B. Rapoport, S.M. McLachlan, Vitamin D deficiency modulates Graves' hyperthyroidism induced in BALB/ $\mathrm{c}$ mice by thyrotropin receptor immunization. Endocrinology 150, 1051-1060 (2009)

6. G. Tamer, S. Arik, I. Tamer, D. Coksert, Relative vitamin D insufficiency in Hashimoto's thyroiditis. Thyroid 21, 891-896 (2011) 
7. H. Yamashita, S. Noguchi, K. Takatsu, E. Koike, T. Murakami, S. Watanabe, S. Uchino, H. Yamashita, H. Kawamoto, High prevalence of vitamin D deficiency in Japanese female patients with Graves' disease. Endocr. J. 48, 63-69 (2001)

8. T. Yasuda, Y. Okamoto, N. Hamada, K. Miyashita, M. Takahara, F. Sakamoto, T. Miyatsuka, T. Kitamura, N. Katakami, D. Kawamori, M. Otsuki, T.A. Matsuoka, H. Kaneto, I. Shimomura, Serum vitamin D levels are decreased and associated with thyroid volume in female patients with newly onset Graves' disease. Endocrine. (2012). doi:10.1007/s12020-012-9679-y

9. N. Vilarrasa, J. Vendrell, J. Maravall, I. Elío, E. Solano, P. San José, I. García, N. Virgili, J. Soler, J.M. Gómez, Is plasma $25(\mathrm{OH}) \mathrm{D}$ related to adipokines, inflammatory cytokines and insulin resistance in both a healthy and morbidly obese population? Endocrine 38, 235-342 (2010)

10. P. Vitti, T. Rago, L. Chiovato, S. Pallini, F. Santini, E. Fiore, R. Rocchi, E. Martino, A. Pinchera, Clinical features of patients with Graves' disease undergoing remission after antithyroid drug treatment. Thyroid 7, 369-375 (1997)

11. K. Khadzkou, P. Buchwald, G. Westin, H. Dralle, G. Åkerström, P. Hellman, 25-Hydroxyvitamin D3 1a-hydroxylase and vitamin
D receptor expression in papillary thyroid carcinoma. J. Histochem. Cytochem. 54, 355-361 (2006)

12. K. Edfeldt, P.T. Liu, R. Chun, M. Fabri, M. Schenk, M. Wheelwright, C. Keegan, S.R. Krutzik, J.S. Adams, M. Hewison, R.L. Modlin, T-cell cytokines differentially control human monocyte antimicrobial responses by regulating vitamin D metabolism. Proc. Natl. Acad. Sci. USA 107, 22593-22598 (2010)

13. M. Rotondi, L. Chiovato, The chemokine system as a therapeutic target in autoimmune thyroid diseases: a focus on the interferon- $\gamma$ inducible chemokines and their receptor. Curr. Pharm. Des. 17, 3202-3216 (2011)

14. A. Antonelli, M. Rotondi, P. Fallahi, P. Romagnani, S.M. Ferrari, L. Barani, E. Ferrannini, M. Serio, Increase of interferon-gammainducible CXC chemokine CXCL10 serum levels in patients with active Graves' disease, and modulation by methimazole therapy. Clin. Endocrinol. 64, 189-195 (2006)

15. T. Kawakami-Tani, E. Fukawa, H. Tanaka, Y. Abe, I. Makino, Effect of 1 alpha-hydroxyvitamin D3 on serum levels of thyroid hormones in hyperthyroid patients with untreated Graves' disease. Metabolism 46, 1184-1188 (1997) 